\title{
Portable three-wavelength standard for energy unit of pulse laser radiation
}

\author{
J. Owsik, A. Zarwalska, Ja. Janucki, V.B. Samoylov* \\ Institute of Optoelectronics, Military University of Technology \\ 2, Kaliskiego St., 00908 Warsaw, Poland, E-mail: jowsik@wat.waw.pl \\ *Institute of Physics NAS of Ukraine, 46, Prospekt Nauki, 03022, Kiev, Ukraine \\ Phone: +380 (44) 26579 52; fax: +380 (44) 26515 89, E-mail: samoylov@iop.kiev.ua
}

\begin{abstract}
New instrument for applications in metrological measurements of laser pulse energy is presented. Due to its parameters, it can be used as a standard for energy unit of pulse laser radiation. The instrument consists of a control unit, three different sources of laser radiation, two detectors of optical signal, and a laptop. The whole system can be easily transported enabling one to perform measurements in situ, at customers, and not only in laboratory conditions. A method of measurements used in the standard operation is described. Main characteristics of the standard are shown. Methods enabling calculations of measurement uncertainties during laser energy meter calibration by means of our standard are also presented.
\end{abstract}

Keywords: laser, energy, metrology, standard, unit.

Paper received 29.12.03; accepted for publication 17.06.04.

\section{Introduction}

In the second half of nineties, a system for metrology protection of laser technique in Poland was suggested [1]. It was established at the Institute of Optoelectronics (IOE) and based on two medium-level stationary standards of power and energy units of laser radiation. Uncertainty of measurements connected to the standard of power unit amounted up to $0.2 \%$, while for the standard of energy unit $-0.85 \%$. In 1998 , a new idea of laser metrology protection system in Poland appeared, grounded on primary standard for radiant power unit based on the absolute cryogenic radiometer [2]. The radiometer with a very low uncertainty (expanded uncertainty for $k=2$ equals $0.05 \%$ ) of measurement has been recently installed at the Central Office of Measures (COM). This system for transfer of units of radiative energy and power, is similar to systems developed earlier in other leading centres dealing with radiative calibration of heat-flux detectors (the Physikalisch-Technische Bundesanstalt - PTB, Germany, and the National Institute of Standards and Technology - NIST, the United States). Both of these systems [3-5] use high accuracy cryogenic radiometers as primary standards for calibrations systems. In both laboratories, the trap detectors, well-known and described elsewhere [6], form the next level of the calibration chain.
The same solution has been adapted to our system (in [7] details of common calibrations of Polish (COM and IOE) and Swedish trap detectors against similar cryogenic radiometer mounted at SP Institute in Bors, Sweden are presented). Subsequent stages of the calibration chain characterised by higher uncertainty of measurement developed at the IOE apparently differ from solutions used in other laboratories, since we established two groups of working standards: the local and portable ones. This resulted from the analysis showing that further development of calibration standards for metrology protection system of laser technique will be focused on three different directions. Those should be: microwatt standards for telecommunication, powerful kilowatt standards for industry, and mobile compact medium-level standards for units of energy or power of laser radiation for any application. The present work deals with such a small, compact, mobile standard of unique capabilities intended for laser energy measurements with reasonably low uncertainty of measurements.

\section{Structure of the standard}

As it has been stated in the previous section, the instrument should meet all the most common demands, as far as the metrology protection system for laser technique is 


\section{J. Owsik et al.: Portable three-wavelength standard for energy unit of pulse laser radiation}

considered. If so, it has to ensure calibration or testing at wavelengths mostly used in laser devices applied in industry, medicine, military applications, and science as well. That is why the standard has been equipped with $\mathrm{Nd}$ :YAG laser $(\lambda=1.06 \mu \mathrm{m})$, frequency converter doubling Nd:YAG frequency $(\lambda=0.53 \mu \mathrm{m})$, and erbium la$\operatorname{ser}(\lambda=1.54 \mu \mathrm{m})$. Two calorimetric measuring transducers have been chosen as radiation detectors. These energy meters have flat spectral characteristics and they withstand high energy levels of input signals. The standard is computer controlled and a special software packet has been prepared for its operation. Optical scheme of the standard is shown in Fig. 1.

\section{Measuring transducer}

Structural arrangement of the calorimetric measuring transducer is presented in Fig. 2. It consists of two calorimetric receivers: the working and compensation ones. Each receiver consists of nickel-deposited copper coneshape cavity with substitution manganin winding assigned for transducer calibration, copper compensation cone, and copper-constantan thermopiles. Both receivers are built in a passive thermostat mounted in two metallic housings. In front of the receivers, a diaphragm is placed, which reduces influence of convection flows and makes transducer operation more reliable.

Principle of operation of the measuring transducer is based on transformation of laser radiation energy into thermoelectromotive force, which is proportional to optical energy. The measured radiation enters working cone 2, which is heated up. This heat is then transmitted to the compensation cone 4. Heating of the compensation cone is registered by thermopiles 5, "hot" outputs of which are placed on the cone 4 , while "cold" ones - into thermostat 11.

The thermopiles are connected according to a differential scheme. Output signals from them are then delivered to an amplifier and gained about $10^{3}$ times. Electrical scheme of the transducer enables it to precise electrical calibration, which should be performed once a year. Electrical scheme of MT-1 transducer is shown in Fig. 3.

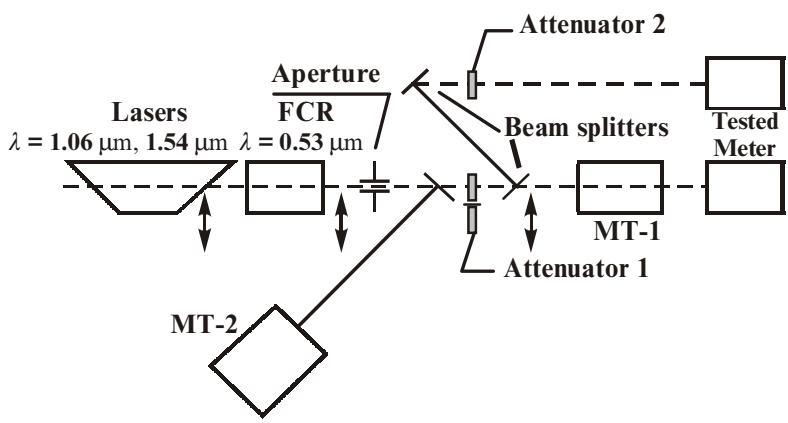

Fig. 1. Optical layout of the standard; MT-1, MT-2 - calorimetric measuring transducers, FCR - frequency converter.

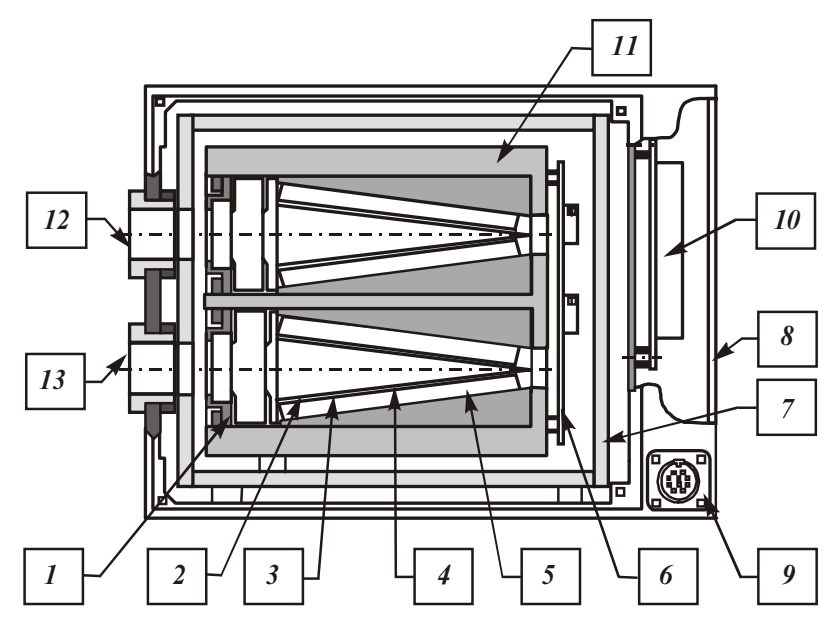

Fig. 2. Calorimetric transducer structure; 1 - diaphragm, $2-$ receiving cone, 3 - substitution winding, 4 - compensation cone, 5 - thermopiles, plate with resistors, 7 - inner housing, 8 - outer housing, 9 - socket, 10 - processing \& control plate, 11 - passive thermostat, 12 - input window of compensating receiver, 13 input window of working receiver.

The measuring transducer has been investigated in order to estimate damage thresholds due to over-exposure of laser radiation. Apart from laser energies, two factors have been taken into consideration, i.e. beam diameter and laser pulse duration. Measurements were carried out using $\mathrm{Nd}$ lasers. Results of measurements are shown in Fig. 4. The chart should be read as follows: if, during measurement, values are too large (high laser energies) or too low (short pulses or small beam diameters) as compared to depicted values in the graph - errors and uncertainties related to measurements will exceed allowed levels for a specified type of the instrument. In some extreme cases, hard damage and disorder of the calorimeter can happen.

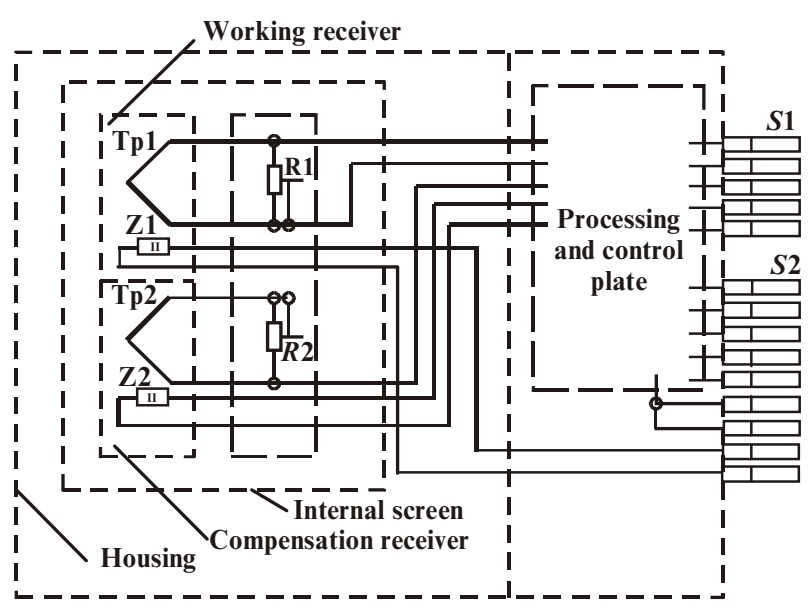

Fig. 3. Electrical scheme of MT-1 transducer. 


\section{Operation of the standard}

Firstly, if the instrument is to operate as a standard for energy unit of laser radiation, it has to receive this energy value from a standard of much less uncertainty of measurements. In our case, MT-1 transducer is given an energy unit from primary standard of mean power and energy unit of laser radiation. Energy unit is given in the form of coefficient of equivalence between laser energy for the wavelength $0.5 \mu \mathrm{m}$ and electrical energy of approximately the same value. Calibration method applied here is based on commonly used electrical substitution of optical energy (electrical and optical heats are compared). This value of energy unit is then being stored for a period of 12 months after which the next calibration must be performed. Of course, for metrological purposes, the calibration chain should be assured, and, if a suitable standard is not available, the so-called intercomparisons are to be carried out.

Typical proceeding aimed at calibration of an energy meter can be divided into some steps, each of them being computer controlled. At the beginning, a proper configuration of the standard must be chosen (operator has to install a laser of the specified wavelength). After 15-minute warming up the standard, its auto-calibration starts. As a rule, it comprises electrical calibration and optical one. In these processes, calorimetric transducers, MT-1 and MT-2, are firstly electrically calibrated. The electrical equivalence factor is found. In order to decrease uncertainty, this calibration can be repeated several times. Optical calibration occurs in the next step, and operator can proceed it up to 8 times. As a result, MT-2 transducer becomes calibrated against MT-1 one - optical equivalence factor between these two transducers is found. The auto-calibration procedure is then completed, and the next MT-1 transducer is removed from the standard configuration.

MT-1 calorimeter is now replaced with unknown tested laser energy meter, which is to be calibrated. Laser pulses in the specified energy range are subsequently generated, and calibration starts. The calibration is performed using MT-2 readings, which are then compared to the readings of the tested meter. The number of comparisons depends only on an operator, but typically it equals to 10 . The ten-fold repetition of measurements makes the uncertainty of calibration more reliable. The calibration factor and its uncertainty as well as the relative error of the calibrated energy meter and its uncertainty are the final results of this calibration procedure.

\section{Main operational and metrological charac- teristics of the standard}

Operational capabilities of the instrument are very interesting, because there are three laser sources used in the standard, calibration can be carried out in several energy ranges of the lasers, and MT- 1 and MT- 2 calorimeters are not spectrally selective (their spectral charac- teristics are flat from 0.4 to $2 \mu \mathrm{m})$. Main operational characteristics are shown in Tables 1,2 , and 3 . The general view of the instrument is presented in Fig. 5.

As it can be seen from Fig. 5, the standard, as a whole, is a medium-sized compact instrument (view of a laptop makes this statement evident). The standard can be dismounted into parts, then put into the three normal-sized cases and easily transported elsewhere. The total weight of the instrument does not exceed $40 \mathrm{~kg}$.

Apart from operational parameters, metrological characteristics of the instrument are also very important. The inherent standard uncertainty of measurements with this instrument, $u\left(E_{S S E}\right)$, resulting from and related to a process of energy unit transfer from the higher level standard and connected to standard readings equals $1 \%$.

As it was mentioned above, the calibration factor $(\mathrm{CF})$ and the relative error $\delta$ of energy meter under calibration (EM) together with combined standard uncertainties of these quantities $u_{C}(\mathrm{CF})$ and $u_{C}(\delta)$ are final results of the calibration process. Methods to calculate these uncertainties and errors were elaborated according to [8].

Errors being made during measurements are sources of uncertainties of CF factor and the relative error $\delta$ as well. Estimation of uncertainties of measurements is performed via two ways: $A$-type estimation - statistical analysis of single measurement results series, and $B$-type estimation - with use of other than statistical methods (for example on the base of catalogue data, supplier data, calibration certificate and so on). Typical procedure, when calculating uncertainties of the factor $\mathrm{CF}$ and the relative error $\delta$, needs some formulae to be applied and they are shown below, after some definitions:

$E_{E M}^{i}$ is EM reading in the $i$-th measurement,

$E_{S S E}^{i}$ is reading of the standard in the i-th measurement,

$n$ is the number of measurements during calibration,

$\bar{E}_{S S E}$ is arithmetic mean of the standard readings,

$\bar{E}_{E M}$ is arithmetic mean of EM readings,

$u_{A}(C F)$ is $A$-type standard uncertainty of CF,

$u_{B}(C F)$ is $B$-type standard uncertainty of $C F$,

$u_{A}(\delta)$ is $A$-type standard uncertainty of the error $\delta$,

$u_{B}(\delta)$ is $B$-type standard uncertainty of the error $\delta$,

$u_{C}(C F)$ is combined standard uncertainty of $\mathrm{CF}$,

$u_{C}(\delta)$ is combined standard uncertainty of $\delta$,

Table 1. Characteristics of the standard equipped with Nd:YAG laser

\begin{tabular}{|l|c|c|c|}
\hline \hline Wavelength, $\mu \mathrm{m}$ & \multicolumn{3}{|c|}{1.064} \\
\hline Energy range, $\mathrm{mJ}$ & $3 \ldots 5$ & $60 \ldots 75$ & $150 \ldots 180$ \\
\hline Beam diameter, $\mathrm{mm}$ & \multicolumn{3}{|c|}{3} \\
\hline $\begin{array}{l}\text { Energy density } \\
\text { in laser beam, } \mathrm{J} / \mathrm{cm}^{2}\end{array}$ & 0.05 & 0.85 & 2.10 \\
\hline Pulse duration, $\mathrm{ns}$ & \multicolumn{3}{|c|}{40} \\
\hline
\end{tabular}




\section{J. Owsik et al.: Portable three-wavelength standard for energy unit of pulse laser radiation}

Table 2. Characteristics of the standard equipped with Nd:YAG laser and frequency converter

\begin{tabular}{|l|c|c|}
\hline \hline Wavelength, $\mu \mathrm{m}$ & \multicolumn{2}{|c|}{0.53} \\
\hline Energy range, $\mathrm{mJ}$ & $10 \ldots 15$ & $40 \ldots 55$ \\
\hline Beam diameter, $\mathrm{mm}$ & \multicolumn{2}{|c|}{3} \\
\hline $\begin{array}{l}\text { Energy density } \\
\text { in laser beam, } \mathrm{J} / \mathrm{cm}^{2}\end{array}$ & 0.15 & 0.55 \\
\hline Pulse duration, $\mathrm{ns}$ & \multicolumn{2}{|c|}{40} \\
\hline
\end{tabular}

$u\left(E_{E M}\right)$ is standard uncertainty of EM readings $u\left(E_{S S E}\right)$ is standard uncertainty of SSE readings,

$\delta E_{E M}$ is resolution of EM readings (from EM manual).

The set of necessary formulae leading to calculation of the calibration factor and relative error as well as to their uncertainties is as follows:

Arithmetic means can be derived as:

$$
\begin{aligned}
& \bar{E}_{S S E}=\frac{1}{n} \sum_{i=1}^{n} E_{S S E}^{i}, \\
& \bar{E}_{E M}=\frac{1}{n} \sum_{i=1}^{n} E_{E M}^{i} .
\end{aligned}
$$

The calibration factor $\mathrm{CF}$ :

$$
C F=\frac{1}{n} \sum_{i=1}^{n} \frac{E_{S S E}^{i}}{E_{E M}^{i}}
$$

Standard uncertainty of EM readings:

$$
u\left(E_{E M}\right)=\frac{\delta E_{E M}}{\sqrt{3}} .
$$

$A$-type standard uncertainty of the calibration factor $\mathrm{CF}$ :

$u_{A}(C F)=\sqrt{\frac{1}{n(n-1)} \sum_{\mathrm{i}=1}^{\mathrm{n}}\left(\frac{E_{S S E}^{i}}{E_{E M}^{i}}-C F\right)^{2}}$. $\mathrm{CF}$ :

$B$-type standard uncertainty of the calibration factor

Table 3. Characteristics of the standard equipped with erbium laser

\begin{tabular}{|l|c|c|l|}
\hline \hline Wavelength, $\mu \mathrm{m}$ & \multicolumn{3}{|c|}{1.54} \\
\hline Energy range, $\mathrm{mJ}$ & $5 \ldots 10$ & $40 \ldots 50$ & $70 \ldots 90$ \\
\hline Beam diameter, $\mathrm{mm}$ & \multicolumn{3}{|c|}{4} \\
\hline $\begin{array}{l}\text { Energy density } \\
\text { in laser beam, } \mathrm{J} / \mathrm{cm}^{2}\end{array}$ & 0.06 & 0.32 & 0.55 \\
\hline Pulse duration, $\mathrm{ms}$ & \multicolumn{3}{|c|}{1.5} \\
\hline \hline
\end{tabular}

SQO, 7(2), 2004

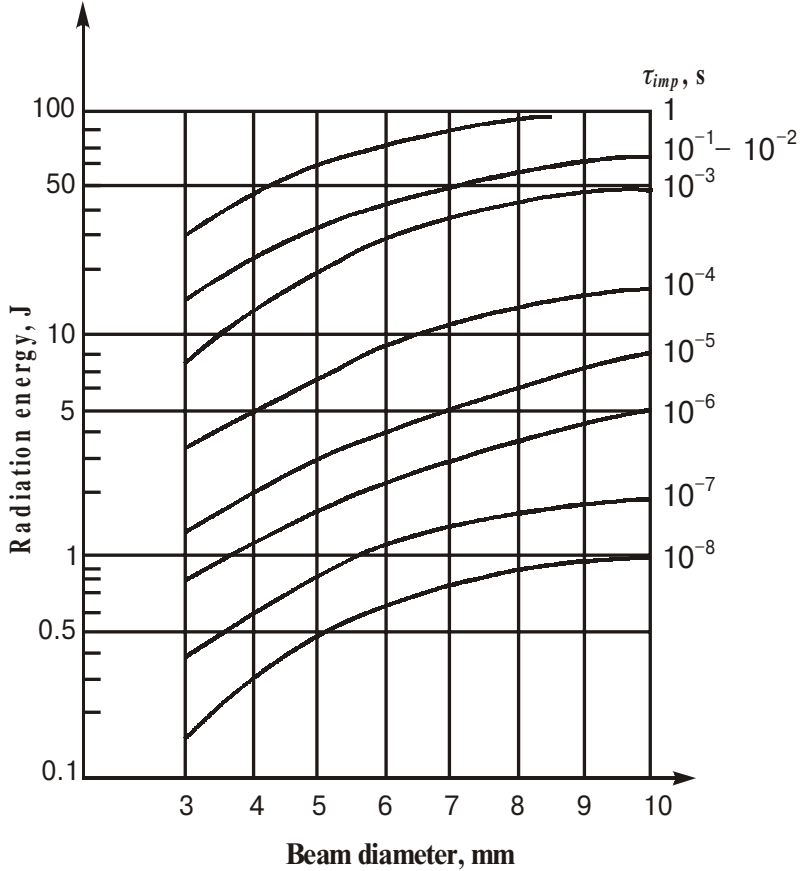

Fig. 4. Maximum allowed values for laser radiation energy versus beam diameter and pulse duration.

$u_{B}(C F)=$

$=\sqrt{\left[-\frac{\bar{E}_{S S E}}{\left(\bar{E}_{P P E}\right)^{2}}\right]^{2} \cdot\left[u\left(E_{P P E}\right)\right]^{2}+\left(\frac{1}{\bar{E}_{P P E}}\right)^{2} \cdot\left[u\left(E_{S S E}\right)\right]^{2}}$.

Combined standard uncertainty of the factor $\mathrm{CF}$ :

$u_{C}(C F)=\sqrt{\left[u_{A}(C F)\right]^{2}+\left[u_{B}(C F)\right]^{2}}$.

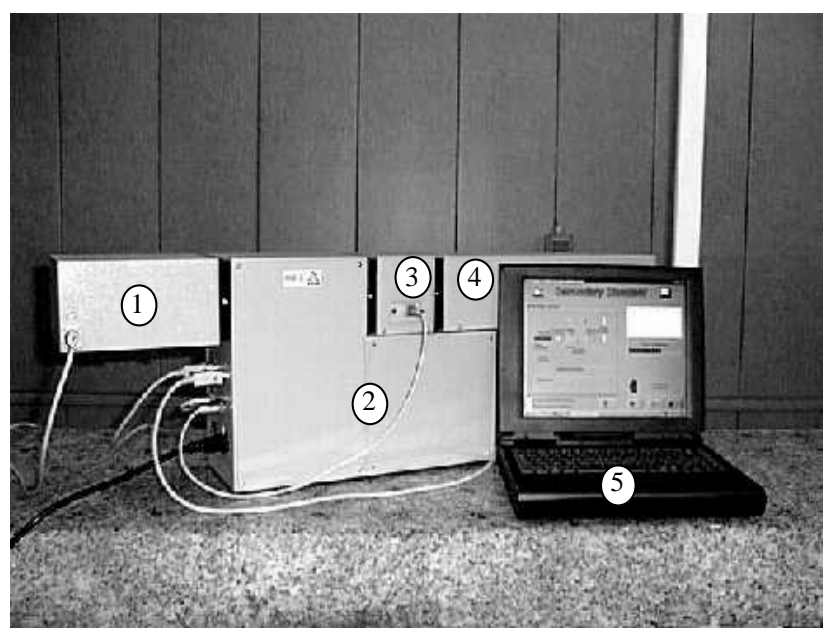

Fig. 5. A picture of the standard for energy unit of pulse laser radiation; 1 - MT-1 calorimetric transducer, 2 - main body of the standard comprising electronics, optical tract, and MT-2 calorimetric transducer, 3 - frequency converter, 4 - Nd:YAG laser, 5 - laptop with main menu of the software. 
Relative error of EM:

$\delta=\left[\frac{1}{n} \sum_{i=1}^{n}\left(\frac{E_{E M}^{i}}{E_{S S E}^{i}}-1\right)\right] \cdot 100 \%$. lows:

$A$-type standard uncertainty of the error $\delta$ is as fol-

$u_{A}(\delta)=\left[\sqrt{\frac{\sum_{i=1}^{n}\left(\frac{E_{E M}^{i}}{E_{S S E}^{i}}-1-\frac{\delta}{100 \%}\right)^{2}}{n(n-1)}}\right] \cdot 100 \%$.

$B$-type standard uncertainty of the relative error :

$u_{B}(\delta)=$

$=\left\{\sqrt{\left(\frac{1}{\bar{E}_{S S E}}\right)^{2} \cdot\left[u\left(E_{E M}\right)\right]^{2}+\left[-\frac{\bar{E}_{E M}}{\left(\bar{E}_{S S E}\right)^{2}}\right]^{2} \cdot\left[u\left(E_{S S E}\right)\right]^{2}}\right\} \times$

$\times 100 \%$.

Combined standard uncertainty of relative error $\delta$ :

$u_{C}(\delta)=\sqrt{\left[u_{A}(\delta)\right]^{2}+\left[u_{B}(\delta)\right]^{2}}$

Based on these formulae, calibration factors and relative errors of any laser energy meters being calibrated against this standard can be found.

\section{Conclusions}

The instrument can be used as the metrological standard in calibration of energy meters for the lasers of $1.06 \mu \mathrm{m}$, $0.53 \mu \mathrm{m}$, and $1.54 \mu \mathrm{m}$ wavelengths, respectively. The latter wavelength is eye-safe one. Generally, the instrument can be used for checking energy meters at any wavelength within the range 0.40 to $2 \mu \mathrm{m}$. In the process of energy unit transfer, it uses a comparative method.

\section{References}

1. J. Owsik, A basis for metrological protection of energy laserometry // J. Techn.Phys., 38(1), pp. 97-109 (1997).

2. J. Pietrzykowski, Primary standard for radiant power based on cryogenic radiometer (in Polish), in Proceedings of Polish National Metrology Congress, KKM'98, Gdansk. 15-18.09.1998.

3. K.D. Stock and H. Hofer, Present state of the PTB primary standard for radiant power based on cryogenic radiometry // Metrologia, 30, pp. 291-296 (1993).

4. T.R.Gentile et al., Calibration of a pyroelectric detector at $10.6 \mu \mathrm{m}$ with the National Institute of Standards \& Technology high accuracy radiometer // Appl.Opt., 36(16), pp. 36143621 (1997).

5. A.V.Murthy and B.K. Tsai and R.D. Saunders, Radiative calibration of heat-flux sensors at NIST: facilities and techniques // Journal of Research of the National Institute of Standards and Technology, 105(2), pp. 293-305 (2000).

6. J. Pietrzykowski, Self-calibrated photodiodes and their applications, in Proc. of optoelectronics Metrology Conf., Lancut, Poland, 28-30.09.1998.

7. G. Werner and L. Liedquist, Calibration of radiant laser power with a cryogenic radiometer at visible wavelengths and at $10.6 \mu \mathrm{m} / /$ SPIE Proceedings, 4018, pp. 73-78 (1998).

8. Guide to the expression of uncertainty in measurement, Geneva, Switzerland, ISO (1993). 